\title{
3D Functional Models of Monkey Brain Through Elastic Registration of Histological Sections
}

\author{
Fabio Bettio ${ }^{1}$, Francesca Frexia ${ }^{1}$, Andrea Giachetti ${ }^{1,2}$, Enrico Gobbetti ${ }^{1}$, \\ Gianni Pintore ${ }^{1}$, and Gianluigi Zanetti ${ }^{1}$ \\ ${ }^{1}$ CRS4 - c/o POLARIS, Edificio 1, Loc. Piscinamanna 09010 Pula (CA), Italy \\ giach@crs4.it \\ 2 Universitá degli Studi di Cagliari, Dipartimento di Matematica e Informatica, \\ via Ospedale, 72, 09124 Cagliari
}

\begin{abstract}
In this paper we describe a method for the reconstruction and visualization of functional models of monkey brains. Models are built through the registration of high resolution images obtained from the scanning of histological sections with reference photos taken during the brain slicing. From the histological sections it is also possible to acquire specifically activated neurons' coordinates introducing functional information in the model. Due to the specific nature of the images (texture information is useless and the sections could be deformed when they were cut and placed on glass) we solved the registration problem by extracting corresponding cerebral cortex borders (extracted with a snake algorithm) and computing an image transform from the deformation linking them. The mapping is modeled as an affine deformation plus a non-linear field evaluated as an elastically constrained deformation minimizing contour distances. Registered images and contours are used then to build 3D models of specific brains by a software tool allowing the interactive visualization of cortical volumes together with the spatially referenced neurons classified and differently colored according to their functionalities.
\end{abstract}

\section{Introduction}

The study of anatomy and functionality of the cerebral cortex is one of the most important research fields in neuroscience. Non-invasive methods to study human brains still have strong limitations in spatial and temporal resolution and in the possibility of correlating anatomical and functional data. Neuroscientists are therefore forced to analyze histological sections of animal brains to understand more precisely morphology and functionality. A typical method to acquire data is the slicing of the frozen brain mass creating histological sections placed on glass plates. From these sections it is possible to recover the position of neurons involved in particular activities that can be visualized on the slices through the use of different tracers. To recover 3D models and locate neurons in a 3D spatial reference it is, however, necessary a complex processing of the acquired data involving non rigid registration of images. To visualize in an effective way the 
data is then necessary to use advanced visualization tools reconstructing surfaces from registered image stacks and rendering them with neuron information added.

In this paper we describe specific registration algorithms and tools for model visualization realized in the framework of the URBAN project, funded by the Italian Ministry of University and Research aimed at the reconstruction of this kind of functional models. Through the use of an ad hoc image registration method and modern visualization techniques, the project allowed neuroscientists to have a clear view of spatially referenced activated neurons over the specific morphology of the brain cortex. Adding cortex segmentation, they can also visualize selectively different functional regions of the brain and different kinds of activated neurons.The paper is organized as follows: Section 1 describes the image registration problem, Section 2 presents the approach chosen, Section 3 the registration results, Section 4 model reconstruction and visualization, Section 5 a short discussion.

\section{Reconstruction Framework}

Image processing techniques can then be used to recover functional models from these glass plates. Digital images can, in fact, be recovered from them with high resolution planar scanners and, being known the slice spacing, voxelized or surface models of the brain with referenced neurons can be built from image stacks. The problem is that the locations of corresponding structures in consecutive images of a stack are not correctly matching because the manual procedure of slice fixation makes slices shifted and deformed by local stretchiness. In order to realign and deform correctly the images and recover therefore the correct spatial location of all the brain structures, digital photos in a fixed reference system are taken. For each glass plate a corresponding photo is thus available and registering the glass plates images with it allows the reconstruction of morphological and functional models of the brain. The reconstruction problem is therefore mainly a non rigid image registration problem, where the main difficulty is due to the lack of texture correspondence between images: only feature points or contours can be used.

\section{Slices Registration}

\subsection{Image Registration: An Overview}

Image registration is a typical task of computer vision. It consists in finding a correspondence map between a pair of images, similarly to what happens in optical flow or stereo matching problems. Here, however, the two images usually represent a $2 \mathrm{D}$ textured planar surface, not a collection of $3 \mathrm{D}$ objects in space, and the correspondence field to be found represents a deformation of the object seen - due to the use of different imaging modalities or, e.g., inter-subject difference in the anatomy of the tissue imaged - rather than the composition of camera motion and object displacement. 
The deformation field linking images acquired using different imaging modalities are, usually, global and with a simple structure, i.e., can be described as affine or polynomial transforms that depend only on changes in sensors orientation and positions and that can be easily identified by imposing the correspondence between few points in the images. Several techniques have been proposed to find points matching, ranging from invasive methods based on the insertion of fiducial markers during the acquisition process, to computer vision algorithms such as landmark recognition, correlation. A complete classification of registration methods used in medical image processing can be found in [7]. In [1] a comparison of results is also presented and a more recent survey can be found in 12. Things are much more complex when one wants to find how to link images of things that are actually different even if related, like corresponding structures of different subjects, stretched objects, etc. This is the case for example of intersubject registration of CT or MRI sections acquired from different patients or intra subject scans at different times and it is also the case of our interest. A typical approach for this task is to apply an iterative elastic deformation [310], driven by the correspondence of control points "recognized" in the two images. Other methods found in literature are piecewise linear transforms [9], or physically based deformations, recently improved by Hagemann et al. [5]. Impressive results have been obtained by Chu and Rangarajan [4 using an iterative method based on a Thin Plate Spline deformation model. The method is fast and robust because of soft-assignment of points correspondence and the possibility of automatically detecting and discarding outliers. Contour based image registration is, however, a slightly different problem, due to the fact that there is no gray level correspondence between the two images and the input of the algorithm are couple of corresponding chains or parametric curves in the two images. The few existing approaches specific to this problem are based on contour points sampling and matching and subsequent point based registration.

A simple algorithm was proposed in [6]. It consists in resampling the images at the same resolution and extract contours as chain codes. Point matching is performed as contours pixel matching from minimization of chain codes' difference. A more advanced approach that is closer to ours was proposed by Davatzikos et al. 2]. They first extract contours from the two images with snakes, then find corresponding points and use an iterative Gauss-Seidel method to solve a dynamical system where the image pixels are shifted driven by the contour points correspondence and by elastic forces (using a spring-mass model) avoiding pixel position folding. The method has problems in handling large deformations and can have problems due to local contour deformations.

\subsection{The Method Implemented: Contour Driven Iterative Deformation}

The method we designed uses of a three step transform handling large deformations and accounting for local contour stretching. Before describing algorithms, let us give a formal description of the problem. We have a target image $T$, and an input image $I$. Finding the correspondence of one or more contours extracted in 
$T, c_{T}$ with contours in $I, c_{I}$, we want to recover the non-linear transform giving for each pixel $p=(i, j)$ in $I$, the corresponding pixel $P=(U(i, j), V(i, j))$ in the reference system of $T$, creating a new image $R$ that we call the "registered" image. Corresponding contours are closed parametric curve $c_{T}(k, s), c_{I}\left(k, s^{\prime}\right)$ where $\mathrm{k}$ is the number of the corresponding contour and $\mathrm{s}, \mathrm{s}$ ' the contour parameter, that in the discrete approximation correspond to the contour point label. The correspondence of contours' points is supposed to drive the image deformation that maps $c_{I}(k, s)$ into $c_{T}(k, s)$ for each $\mathrm{k}, \mathrm{s}$.

Contour Extraction. Contours are extracted from raster images with a snake algorithm [8]. In our implementation contours are simple chains of points. Forces driving their iterative evolution are: standard elastic/rigid forces (computed with finite differences), an inflating or deflating force making the curve move toward the region limits, image force based on an RGB threshold (contour evolution is stopped if the distance of the color value from the central on in the RGB space is lower than a fixed threshold), a color edge force. Automatic resampling keeps the point distribution constant (the number of points or the point spacing are kept constant). In our experiments, functional data were often acquired with another method, i.e. using a particular device allowing neuroscientists to inspect through a microscope glass plates and to manually trace borders and mark neuron positions. Contours' and neurons' coordinates were in these cases given us as a vector image describing the contours as polylines and neuron positions as $2 \mathrm{D}$ coordinates. In this case the functional model can be recovered in the same way, registering the functional data with respect to the same reference photographic images. The registration procedure for this data is the same,only the contour to be mapped on the reference image is directly selected on the file instead of computed with the snake and the transform obtained is applied then to the neurons instead of to the pixel array.

Point Matching. When couples of corresponding contours are found, we resample them with the same number of point, equally spaced $P_{1}(i), P_{2}(i)$. Then we match labeled points with a nonlinear transform considering the possibility of local stretching of the contours. The procedure adopted is the following: first a global correspondence of the original equally spaced nodes of the original contour parameterization is done by finding the integer label shift of the point of $P_{2}, s$ minimizing the function: $f=\sum_{i=1}^{N}\left(w\left(P_{1}(i)\right)-w\left(P_{2}((i+s) \bmod N)\right)\right)^{2}$, where $w$ is a function describing locally the curve not depending on the reference system. We took our $w$ as a linear combination of the squared distance of the point from the center of mass of the curve divided by the surface enclosed by the curve and the local curvature. Then feature points are detected in the contours extracted from the target and the input images. If two feature points of the same kind are found among the points of the input and target contours within a small label range, a correspondence between the two points is set. When all the corresponding features are found, the input contour is resampled giving the same label to the corresponding feature point and resampling the two contours between consecutive features points with the same number of equally labeled points. Features considered are: 
-Local maxima of curvature larger than a threshold.

-Local minima and maxima of the distance from the center of mass.

Linear Transform. The first step in the registration procedure consists of a linear transformation. Writing its equation for points of corresponding contours with the same label an overconstrained system is obtained, and its least-squares solution gives the best linear transformation mapping the input image pixels into the target image pixels. The linear transform parameters are saved as well as the displacement field mapping the input image into the linearly registered one. The field and the new image are built by creating a pixel grid of target image size and computing for each pixel location the vector mapping the target pixel into the input image. A bilinear interpolation is finally used to compute the color components of the pixel from the four input image pixels closest to these non-integer transformed coordinates.

Fast Iterative Elastic Transform. A non linear correction is used as the second registration step. It is an iterative elastic method with some features in common with the method used in [2] The basic idea is that pixels are supposed to be joined by springs to their 4 neighbors keeping them close, while pixels corresponding to matching contours are shifted according to the field defining the contours correspondence. The input image is the linearly registered one and the target image is the same as before. We create then a registered image of the target image size. The goal is to put in each pixel of this image values of the linearly registered one at a transformed location. The pixel transformations are computed through an iterative algorithm (similar to the one described in [2]) minimizing with a greedy algorithm the elastic potential with the constraints of zero motion at image borders and reassigning periodically input shifts at contour pixels. To compute these shifts we apply a Bresenham rasterization of the target contour and we assign to each pixel obtained a shift vector equal to its distance from the linearly registered corresponding contour. To speed up the computation, shifts of pixels inside the contour are not initialized as null vectors, but with $\mathrm{x}(\mathrm{y})$ values equal to a weighted average of the two closest border shifts along the $\mathrm{x}(\mathrm{y})$ direction. Weights are inversely proportional to the corresponding border points distance.

This procedure initializes the system closer to the equilibrium position. Finally pixel shifts is iteratively calculated from the spring-mass system reassigning periodically the distance values to the border pixels. Iterations are stopped when non border pixel shifts are lower than a fixed threshold.

\section{Experimental Results}

Tests on Registration. We first applied registration algorithms on two test image pairs, the first simulating a linear deformation and the second adding to a known linear transform some local non-linear effects similar to those coming from the acquisition of a histological sample (i.e with local stretching and irregularities). Recovered and ground-truth displacement fields have finally been compared 


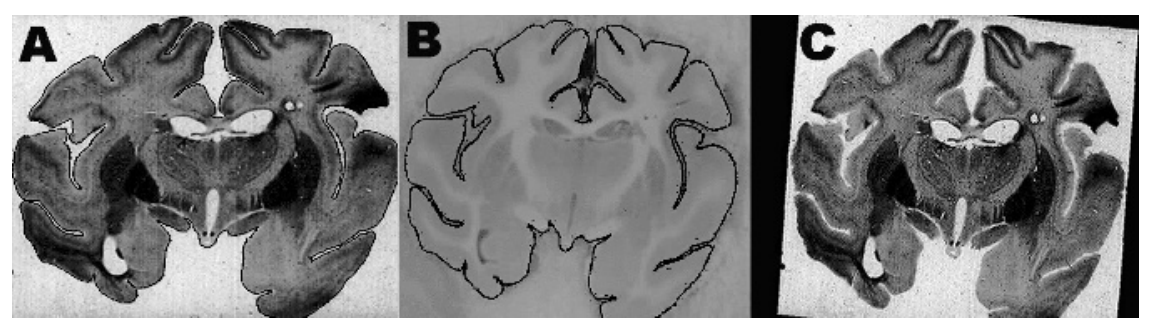

Fig. 1. Results on a real image pair. A,B: input and target images (and superimposed contour in black). C: registration results.

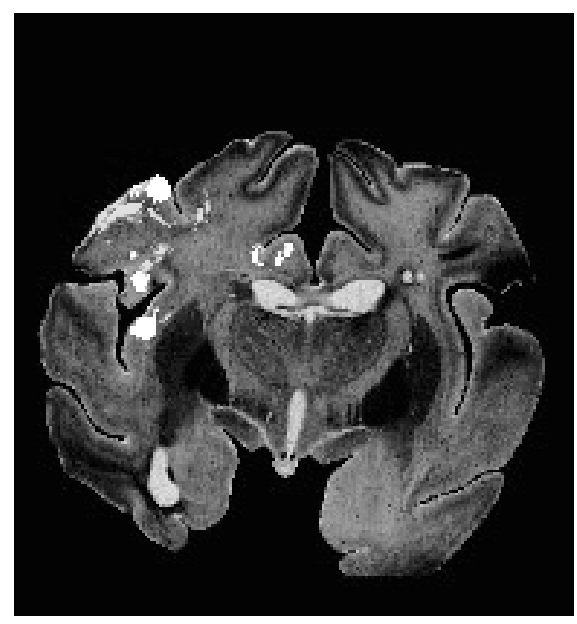

Fig. 2. Image representing more than 4000 classified neurons (bright spots) from a microscopy acquisition mapped over the registered slice texture

with standard optical flow difference measures [1] with the displacement fields computed by the registration algorithms. Experiments on the linearly deformed pattern have shown the advantages of using the feature based contour point sampling instead of the uniform contour sampling used in [2]. The average error in the deformation field for the internal pixels of the shape is decreased from an average unsigned difference of 1.3 to 0.3 and from an average Barron angular distance of 0.5 to 0.1 , i.e. there is a great improvement in the registration accuracy due to the feature based resampling step. Tests performed also on the nonlinear synthetic deformation showed then that the minimization of point/contour distances provides better results than the minimization of point-point distances.

Registration of real images with our final method obtain qualitatively good results. Fig. 1A shows a reference photo acquired during the sectioning of a frozen monkey brain. Fig. 1B shows the corresponding scanning of the histological section. Contours semi-automatically recovered with deformable contours are represented superimposed to the corresponding image. It must be noted that 

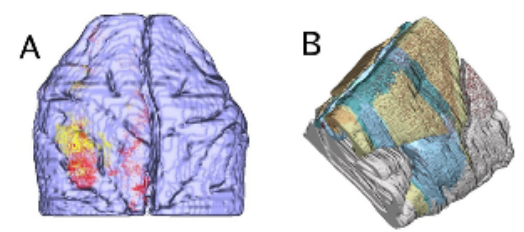

Fig. 3. Left: Brain reconstruction rendering with two neuron classes displayed in different colors. Right Brain reconstruction rendering with seven functional cortex section painted in different color and only one neuron class displayed.

the quality of the scans is not always satisfactory, so that automatically computed contours should be in some cases checked and locally deformed through a user friendly interface. Couple of corresponding images and contours is then registered with the method described in order to build the morphological brain model. Fig.1C shows the results of the registration algorithm based on linear deformation and fast elastic adaptation. Every histological section has been acquired at a resolution of $600 \mathrm{dpi}$ which corresponds to an actual pixel size of about 70x70 microns. Neurons were acquired with the microscopy tool at University of Parma. Fig.2 shows an example of registration of classified neurons mapped over the corresponding slice textures. Both neurons and texture are mapped with the presented technique over the reference photos coordinates.

Model Reconstruction and Visualization. From the realigned images it is possible to build precise voxelized or surface models of the brain (linking borders in consecutive slices). We implemented an user friendly interface allowing the visualization of $3 \mathrm{D}$ reconstructed brain models. The main operations performed by the system are model reconstruction, using different algorithms such as isosurface extraction or point splatting technique, and multipass rendering, to display interactive selected ranges of labeled cortical neurons. Figure 3A shows a reconstructed brain cortex surface, rendered in an user friendly interface proving the neuroscientists useful tools for the functional analysis. Spatially referenced neurons are represented as dots painted through a colormap representing its functionality (here we see two types in red and yellow). The interactive tool allows the user to visualize only selected neurons. Neurons are modeled as emissive particles, while the brain material is considered as a semi-transparent medium with constant absorption coefficient. Absorption is computed based on distance from one neuron to the brain surface closest to the eye. In the case of Fig.3B, different functional regions of the brain have been segmented and represented in different colors, with superimposed neurons of a single type represented in red.

\section{Conclusions}

We presented a framework for the reconstruction of functional models of monkey brain. It is based on a contour based image registration method that is an improvement of other approaches found in literature and on an interactive tool 
for model analysis and visualization. Results have been obtained within the URBAN project, in collaboration with the Dept. of Neuroscience of the University of Parma, aimed at the reconstruction of 3D functional maps of primate brains, funded by the Italian Ministry of Instruction, University and Research.

\section{References}

1. J.L. Barron, D.J. Fleet, S.S. Beauchemin, and T.A. Burkitt. Performance of optical flow techniques. CVPR, 92:236-242.

2. C. Davatzikos, J. Prince, and R. Bryan. Image registration based on boundary mapping. IEEE Transactions on Medical Imaging, 15:112-115, 1996.

3. Matthieu Ferrant, Simon K. Warfield, Charles R. G. Guttmann, Robert V. Mulkern, Ferenc A. Jolesz, and Ron Kikinis. 3d image matching using a finite element based elastic deformation model. In MICCAI, pages 202-209, 1999.

4. A. Rangarajan H. Chui. A new point matching algorithm for nonrigid registration. Comput. Vision Image Und., 89:109-111, 2003.

5. A. Hagemann, R. Stiel, and U. Spetzger. Non-rigid matching of tomographic images based on a biomechanical model of the human head. In SPIE Medical Imaging '99. 1999.

6. H. Li, B.S. Manjunath, and S.K. Mitra. A contour-based approach to multisensor image registration. IEEE Trans on Image Processing, 4(3):320-334, March 1995.

7. J. Maintz and M. Viergever. A survey of medical image registration. Medical Image Analysis, 2(1):1-36, 1998.

8. D.Terzopoulos M.Kass, A.Witkin. Snakes: Active contour models. Int. Journal of Computer Vision, 1:321-331, 1987.

9. A. Pitiot, G. Malandain, E. Bardinet, and P. Thompson. Piecewise Affine Registration of Biological Images. In Second International Workshop on Biomedical Image Registration WBIR'03, 2003.

10. T. PM and T. AW. Elastic image registration and pathology detection, 2000.

11. J. West, J. Fitzpatrick, M. Wang, B. Dawant, C. Maurer, R. Kessler, and R. Maciunas. Comparison and evaluation of retrospective intermodality image registration techniques, 1996.

12. B. Zitova and J. Flusser. Image registration methods: a survey. IVC, 21(11):9771000, October 2003. 